\title{
Olfactory Deficits Cause Anxiety-Like Behaviors in Mice
}

\author{
Meredith E. Glinka, ${ }^{1}$ Benjamin A. Samuels, ${ }^{2}$ Assunta Diodato, ${ }^{4}$ Jérémie Teillon, ${ }^{4}$ Dan Feng Mei, ${ }^{1}$ Benjamin M. Shykind, ${ }^{3}$ \\ René Hen, ${ }^{2}$ and Alexander Fleischmann ${ }^{1,4}$ \\ ${ }^{1}$ Department of Neuroscience and the Howard Hughes Medical Institute, and ${ }^{2}$ Department of Neuroscience and Department of Psychiatry, Columbia \\ University, New York, New York 10032, ${ }^{3}$ Weill Cornell Medical College in Qatar, Qatar Foundation-Education City, Doha, Qatar, ${ }^{4}$ Center for \\ Interdisciplinary Research in Biology, College de France, 75005 Paris, France
}

Anxiety disorders are characterized by persistent fear in the absence of immediate threat and represent the most common psychiatric diseases, with an estimated $28 \%$ lifetime prevalence worldwide (Kessler et al., 2010). While symptoms of anxiety are typically evoked by sensory stimuli, it is unknown whether sensory deficits contribute to the development of anxiety disorders. Here we examine the effect of defined genetic mutations that compromise the function of the olfactory system on the development of anxiety-like behaviors in mice. We show that the functional inactivation of the main olfactory epithelium, but not the vomeronasal organ, causes elevated levels of anxiety. Anxiety-like behaviors are also observed in mice with a monoclonal nose, that are able to detect and discriminate odors but in which the patterns of odor-evoked neural activity are perturbed. In these mice, plasma corticosterone levels are elevated, suggesting that olfactory deficits can lead to chronic stress. These results demonstrate a central role for olfactory sensory cues in modulating anxiety in mice.

\section{Introduction}

The sense of smell is the primary sensory modality for animals to detect food, predators, and mates. Not surprisingly, lesion studies that compromise olfactory function in rodents have broad behavioral consequences. Bulbectomy, the surgical removal of the olfactory bulbs, not only results in anosmia, but also altered sleep patterns and deficits in sexual, aggressive, and maternal behaviors (for review, see Brunjes, 1992). In addition, bulbectomy leads to anxiety- and depression-like behaviors. Because most of the behavioral changes caused by the surgical removal of the olfactory bulbs can be reversed by chronic antidepressant treatment, bulbectomized rats have frequently been used as a model for testing antidepressant drugs (Cairncross et al., 1977; van Riezen and Leonard, 1990; Brunjes, 1992; Kelly et al., 1997; Harkin et al., 2003; Song and Leonard, 2005).

Recent genetic approaches in mice have provided a more detailed understanding of the functions of the olfactory system. The mouse olfactory system comprises two anatomically distinct sensory organs, the main olfactory epithelium (MOE) and the vomeronasal organ (VNO). The nucleotide-binding subunit of the

Received Aug. 20, 2011; revised March 21, 2012; accepted March 23, 2012.

Author contributions: B.A.S., R.H., and A.F. designed research; M.E.G., A.D., J.T., D.F.M., and A.F. performed research; B.M.S. contributed unpublished reagents/analytic tools; M.E.G., B.A.S., A.D., J.T., R.H., and A.F. analyzed data; A.F. wrote the paper.

This work was supported by a long-term postdoctoral fellowship from the Human Frontiers Science Program, a Marie Curie International Reintegration grant, and the Amorcage de jeunes equipes program of the Fondation pour la Recherche Medical (to A.F.). B.A.S. is a Revson Senior Fellow. Work in R.H.'s laboratory is supported by the NIMH Grant R01 MH068542. Behavioral experiments were performed in Richard Axel's laboratory, which is supported by the Howard Hughes Medical Institute. We thank Sunita Patruni, Lyudmila Burina, and Ebru Demir for help with the generation of transgenic mice. We thank Dara Sosulski, Mark Albers, Gilad Barnea, Christoph Kellendonk, Andreas Schäfer, Ken Moya, and Richard Axel for comments on the manuscript.

R.H. receives compensation as a consultant for BrainCells, Inc. in relation to the generation of novel antidepressants.

Correspondence should be addressed to Alexander Fleischmann at the Center for Interdisciplinary Research in Biology, College de France, 75005 Paris, France. E-mail: alexander.fleischmann@college-de-france.fr.

DOI:10.1523/JNEUROSCI.4287-11.2012

Copyright $\odot 2012$ the authors $\quad 0270-6474 / 12 / 326718-08 \$ 15.00 / 0$ cyclic nucleotide gated (CNG) channel, CNGA2, was shown to be essential for odor-evoked signal transduction in the MOE (Brunet et al., 1996). Targeted deletion of the Cnga2 gene in mice results in general anosmia and increased neonatal lethality (Brunet et al., 1996), and adult Cnga2-deficient mice display deficits in sexual and aggressive behaviors (Mandiyan et al., 2005). The targeted deletion of the transient receptor potential cation channel c2 ( $\operatorname{Trpc} 2)$ was shown to impair sensory activation of VNO neurons by pheromones (Leypold et al., 2002; Stowers et al., 2002). Trpc2-deficient mice exhibit defects in gender recognition and aggressive behaviors (Leypold et al., 2002; Stowers et al., 2002; Kimchi et al., 2007). Cnga2 and Trpc2 mutant mice thus provide well characterized genetic models to specifically test the behavioral consequences of the functional ablation of the MOE and $\mathrm{VNO}$, respectively.

Olfactory sensory neurons in the MOE express one of 1300 odorant receptor (OR) genes (Buck and Axel, 1991; Zhang and Firestein, 2002). Mice with perturbations in the expression of the OR gene repertoire can reveal insights into how receptor activation in the periphery is transformed into odor representations in the brain that guide behaviors and provide a complementary model to assess olfactory functions (Nguyen et al., 2007, 2010; Fleischmann et al., 2008). In M71 transgenic mice, the expression of the M71 OR is under the control of the tTA-responsive tet operator $\left(\right.$ tet $\left._{O}\right)$ (Gossen et al., 1995). tTA binding to the tet $_{O}$ results in the activation of the promoter. However, in the presence of the tetracycline-derivative doxycycline (Dox), tTA binding to its target is abolished, allowing for the conditional control of transgene expression. In these mice with a monoclonal nose, $>95 \%$ of sensory neurons express the M71 receptor, which results in perturbed patterns of odor-evoked neural activity (Fleischmann et al., 2008). Exposure to the M71 receptor ligand acetophenone activates the vast majority of sensory neurons and all glomeruli, while odorants that do not activate the M71 receptor do not yield detectable glomerular activity. These perturba- 
tions in odor-evoked neural activity cause severe deficits in innate olfactory-driven behaviors, including mating and aggression. In contrast, odor detection in assays for learned olfactory behaviors appeared largely intact, except for the inability of M71 transgenic mice to detect the M71 receptor ligand acetophenone (Fleischmann et al., 2008).

In this study, we took advantage of the well characterized olfactory deficits of Cnga2 and Trpc2 knock-out and M71 transgenic mice to examine the role of the olfactory system in the development of anxiety- and depression-like behaviors. We observed elevated levels of anxiety in Cnga2-deficient, but not Trpc2-deficient, animals, revealing a central role for the MOE, but not the VNO, in modulating anxiety in mice. In contrast to a large set of previous surgical lesion studies (for review, see Brunjes, 1992), we find that the genetic ablation of olfactory function does not cause elevated levels of depression-related behaviors. Interestingly, we found that M71 transgenic mice with a monoclonal nose exhibit anxiety similar to, but even more pronounced than, those observed in mice with a functional ablation of the MOE. Furthermore, M71 transgenic mice have elevated levels of blood corticosterone. These results suggest that olfactory deficits may cause chronic stress, resulting in the development of anxiety-like behaviors.

\section{Materials and Methods}

Littermate male mice on a mixed $129 \mathrm{SvEv} \times \mathrm{C} 57 \mathrm{BL} / 6$ genetic background were used for all experiments. To obtain $\mathrm{Cnga2}^{-/ Y}$ males, heterozygous females were mated to wild-type males. $\operatorname{Trpc2^{+/-}}$ males and females were bred to generate $\operatorname{Trpc} 2^{-1-}$ males and wild-type controls. Heterozygous OMP-IRES-tTA and hemizygous tet ${ }_{\mathrm{o}}$-M71-IRESlac $Z$ transgenic mice were bred to generate compound heterozygotes OMP-IRES-tTA/tet ${ }_{\mathrm{o}}$-M71-IRES-lacZ transgenic mice (referred to as M71 transgenic mice), and wild-type, OMP-IRES-tTA heterozygous and hemizygous tet ${ }_{\mathrm{o}}$-M71-IRES-lacZ controls. Data from control genotypes were polled since no differences were observed between the three groups (one-way ANOVA).

Mice were housed on a reversed $12 \mathrm{~h}: 12 \mathrm{~h}$ light:dark cycle. All tests were performed in compliance with the Columbia University Institutional Animal Care and Use Committee. Behavioral tests were performed with male mice 10-14 weeks of age, with cohorts no larger than 16 mice. Small differences in the number of mice used in each assay resulted from few mice being able to jump onto the border of the container. These mice were excluded from the analysis. Mice were single-housed for 1 week before testing. Anxiety- and depression-related assays were performed on consecutive days in the following order: open field, light-dark, novelty-suppressed feeding, tail suspension, and forced swim tests. $p$ values reflect unpaired, two-tailed $t$ tests. Significant effects were confirmed by two-sample Kolmogorov-Smirnov tests (ks tests).

Doxycycline treatment. In M71 transgenic mice, the expression of the M71 OR transgene is controlled by the tTA transcription factor expressed from the OMP promoter. The tTA transcription factor activates the tet promoter in the absence of doxycycline, and administration of doxycycline ablates DNA binding and shuts off transgene expression. Doxycyclineinfused food $(200 \mathrm{mg} / \mathrm{kg}$ ) was purchased from BioServe and administered to mice throughout development (through maternal feeding) and postnatally until the completion of the behavioral testing. This Dox-infused food contains more calories than regular rodent chow ( $3.75 \mathrm{vs} 3.4 \mathrm{kcal} / \mathrm{g}$ ), resulting in an $\sim 10 \%$ increase in body weight of mice at 12 weeks of age (data not shown). To control for dietary effects on behaviors, M71 transgenic and control mice were raised on a Dox diet in these experiments.

Open-field test. The open-field assay was conducted in a $47 \times 66 \times 23$ $\mathrm{cm}$ container. Red light, invisible to mice, was used for video tracking purposes. At the start of each trial, the mouse was placed in a corner of the container and its movement was tracked for $30 \mathrm{~min}$. Noldus Ethovision 3.0 tracking software was used to map center and periphery zones and to calculate the total duration, frequency, and latency in the center zone as well as the distance traveled in total and in the center.
Light-dark choice test. The light-dark choice assay was conducted in a container similar to that of the open-field test, but the container was divided in half by black posterboard covering the top half of the container, creating the dark compartment. Regular white light was used to illuminate the light compartment. The trial began when the mouse was placed at the entryway to the dark compartment; the trial duration was 30 min. Ethovision tracking software recorded the total amount of time the animal spent in the light half of the container.

Novelty-suppressed feeding. Mice were food-deprived for $24 \mathrm{~h}$ before testing. The mouse's home cage bedding was sprinkled over the floor of the testing container and a piece of Whatman paper was placed in the center of the container, with rodent chow placed in the center of the paper. The testing room was brightly illuminated with a $100 \mathrm{Watt}$ bulb. The trial duration was $10 \mathrm{~min}$ and the latency to begin feeding (defined as nibbling the food pellet for $>4 \mathrm{~s}$ ) was recorded by an observer blind to the genotype.

Tail suspension test. Mice were suspended upside-down from their tail using adhesive tape for a total duration of $6 \mathrm{~min}$. The session was recorded by a video camera and the total time immobile was scored.

Forced swim test. Forced swim tests were done in a $25 \times 25 \times 35 \mathrm{~cm}$ Plexiglas box filled with room temperature water. The mouse was placed into the water and behavior was recorded for $6 \mathrm{~min}$. The experiment was conducted twice on consecutive days, with only data from the second day analyzed. The total duration of immobility during the last $4 \mathrm{~min}$ of the trial, defined as not moving both the front and back legs, was scored.

Auditory fear conditioning. Mice received three consecutive toneshock pairings ( 3 min apart, $80 \mathrm{~dB}, 2 \mathrm{kHz}$, and foot shocks of $0.5 \mathrm{~mA}$ delivered for $1 \mathrm{~s}$ at the offset of each tone). The next day, mice were tested in a novel environment with new tactile and visual cues. Freezing was recorded manually from videotape recordings, and $3 \mathrm{~min}$ without tone (novel context) and subsequent $6 \mathrm{~min}$ with tone were analyzed. Results were confirmed using automated Noldus Ethovision 3.0 tracking software.

Blood plasma corticosterone. Corticosterone levels were determined using the Corticosterone EIA kit (Assay Design) according to the manufacturer's instructions. Blood was collected from the superficial temporal vein (submandibular) at 1 P.M. for all mice to exclude variability due to circadian fluctuations. Mice used for plasma corticosterone analysis were not subjected to any behavioral tests.

Immunohistochemistry. Analysis of OR expression was performed on 16 $\mu \mathrm{m}$ cryosections of tissue that was fixed in $1 \%$ paraformaldeyde (EMS) and embedded in OCT (Sakura). Anti-lacZ antiserum (Biogenesis) was used at 1:1000 dilution, anti-M50 and anti-M71 antiserum (Fleischmann et al., 2008) were used at 1:2000 dilution. Primary antibodies were visualized using Alexa 488 and Cy3-conjugated secondary antibodies (Invitrogen and Jackson Laboratories) and counterstained with Toto-3, 1:1000 (Invitrogen). Stained sections were visualized using a Zeiss 710 confocal microscope. Analysis of c-Fos expression was performed on a separate group of singlehoused mice in their home cage or after $45 \mathrm{~min}$ in the open-field (OF) arena. Mice were killed and brains were perfusion-fixed with $4 \%$ paraformaldehyde. Brain slices were cut coronally at $200 \mu \mathrm{m}$ and incubated overnight with anti-c-Fos antiserum (1:1000, sc-52-G; Santa Cruz Biotechnology), followed by incubation with anti-goat-Alexa 488 antiserum (1:1000; Invitrogen) and counterstained with NeuroTrace fluorescent Nissl (Invitrogen). Z-stacks, 80 $\mu \mathrm{m}$ in total thickness at bregma -2.3 , were acquired using a Leica SP5 confocal microscope with a $10 \times$ objective. Quantification of c-Fos-positive nuclei of tissue sections obtained from four to six mice per experimental condition was performed in ImageJ software. After background correction, regions of interest [piriform cortex, visual cortex (V1), and somatosensory cortex (S1)] were drawn according to The Mouse Brain Atlas (Franklin and Paxinos, 2007). c-Fos-positive nuclei were defined by a pixel value $>100$, an object size of $50-150$ pixels, and a circularity $>0.2$.

\section{Results}

Genetic ablation of the MOE, but not the VNO, causes elevated levels of anxiety

In the present study, we used the previously generated Cnga2 (MOE mutant), Trpc2 (VNO mutant), and M71 transgenic 

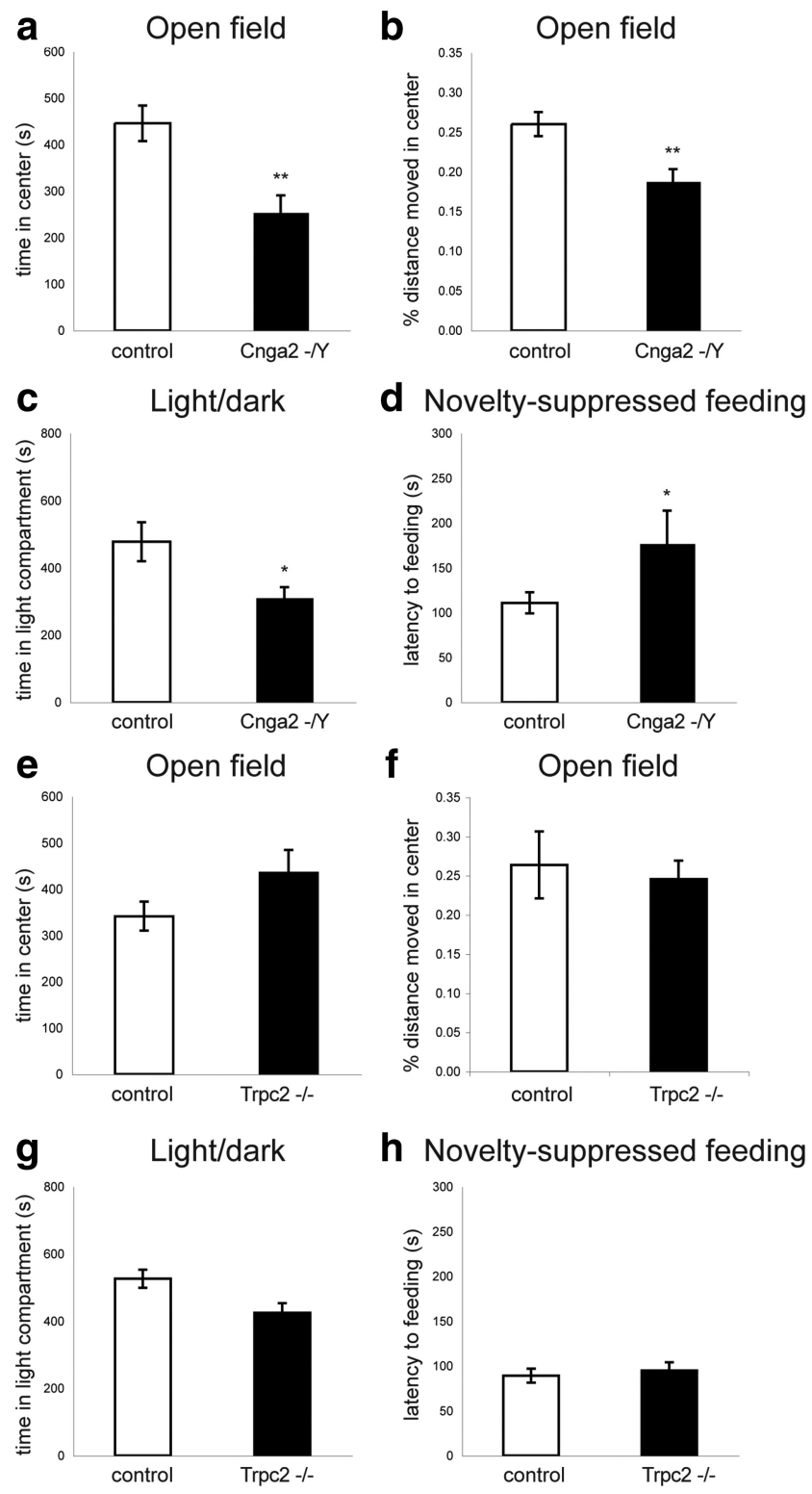

Figure 1. Elevated levels of anxiety in $\mathrm{Cnga2}^{-/ \gamma}$ but not in $\operatorname{Trpc} 2^{-/-}$mice. $\boldsymbol{a}, \boldsymbol{b}$, Open field test. The time spent $(\boldsymbol{a})$ and the percentage of distance moved $(\boldsymbol{b})$ in the center of the open field is reduced in Cnga2 mutant $(n=16)$ compared with controls $(n=20)$. c, Light- dark choice test. Cnga2 mutant mice spent less time in the light compartment compared with controls $\left(C n g a 2^{-N}: n=17\right.$; controls: $\left.n=21\right)$. $\boldsymbol{d}$, Novelty-suppressed feeding. The latency to begin feeding was increased in Cnga2 mutant ( $n=16$ ) compared with control $(n=21)$ mice. $\boldsymbol{e}, \boldsymbol{f}$, № significant differences were observed in the time spent $(\boldsymbol{e})$ and the percentage of distance moved $(\boldsymbol{f})$ in the center of the open field between $\operatorname{Trpc2^{-/-}}(n=18)$ and control $(n=26)$ mice. $\boldsymbol{g}$, The time spent in the light compartment of the light-dark choice test was similar between $\operatorname{Trpc2^{-/-}}(n=18)$ and controls $(n=28)$. $\boldsymbol{h}$, Novelty-suppressed feeding. No significant difference in the latency to begin feeding was observed between $\operatorname{Trpc} 2$ mutant $(n=18)$ and control $(n=28)$ mice. ${ }^{*} p<0.05,{ }^{* *} p<0.01$. Error bars are mean \pm SEM.

mouse lines to test the role of olfaction in the development of anxiety- and depression-like behaviors. We first examined anxiety-related behaviors in Cnga 2 and $\operatorname{Trpc} 2$ mutant mice using the OF test. The OF test is an approach-avoidance task, which exploits conflicting tendencies to explore a novel environment versus avoiding the center of the arena, a potentially dangerous area (David et al., 2009). We found that Cnga2 mutant mice spent significantly less time in the center of the arena than wild-type controls $\left(t_{(34)}=3.50, p<0.002\right.$; Fig. $\left.1 a\right)$. Consistently, the per- centage of distance moved in the center was reduced in Cnga2 mutant mice $\left(t_{(34)}=3.29, p<0.003\right.$; Fig. $\left.1 b\right)$, while the overall distance traveled was unchanged [path length (in m): $\mathrm{Cnga2}^{-/-}$: $13.4 \pm 0.7$; controls: $12.3 \pm 0.3, t_{(34)}=1.59, p>0.1$ ] (values are mean \pm SEM throughout). In contrast, anxiety-related behaviors of $\operatorname{Trp} 22$ mutant mice in the OF were similar to wild-type controls (time spent in center: $t_{(42)}=1.64, p>0.1$; Fig. $1 e$; percentage of distance moved in the center, $t_{(42)}=-0.33, p>$ 0.7 ; Figure $1 f$ ).

In the light-dark (LD) choice test, based on the tendency of mice to avoid brightly illuminated spaces, we found that Cnga2 mutant mice spent significantly less time in the light compartment compared with controls $\left(t_{(36)}=2.36, p<0.03\right.$; Fig. $\left.1 c\right)$. Consistently, the number of light-dark transitions was significantly reduced (LD transitions: Cnga2 ${ }^{-/-}: 64.4 \pm 5.5$; controls: $\left.85.5 \pm 7.4, t_{(34)}=2.21, p<0.04\right)$. The behavior of Trpc2 mutant mice in this assay was again similar to that of controls (time spent in light compartment: $t_{(44)}=1.88, p>0.05$; Fig. $1 g$ ). There was no significant difference between $\operatorname{Tr} p c 2$ mutant and control mice in the number of light-dark transitions $\left(\operatorname{Trp} c 2^{-1-}: 95.9 \pm 4.5\right.$; controls: $\left.90.4 \pm 6.2 ; t_{(44)}=0.73, p>0.1\right)$. The behavioral changes observed in Cnga2-deficient mice are specific to these approach-avoidance conflict tests since general exploratory behavior and locomotor activity in the home cage did not differ between genotypes (data not shown).

We next examined the behavior of MOE and VNO mutant mice in the novelty-suppressed feeding (NSF) paradigm. This test depends less on exploratory behavior and elicits competing motivations between the drive to eat and the fear of venturing into the center of a brightly lit arena. We found that Cnga2 mutant mice exhibited a longer latency to begin feeding compared with controls $\left(t_{(35)}=-2.09, p<0.05\right.$; Fig. $\left.1 d\right)$. This difference was specific to the anxiogenic setting since home cage feeding behavior was indistinguishable between the genotypes (data not shown). In contrast, no differences were observed between $\operatorname{Trpc} 2$ mutant and control mice (latency to begin feeding: $t_{(44)}=0.49$, $p>0.5$; Fig. $1 h$ ). Together, these data reveal that olfactory cues detected by the MOE, but not the VNO, play an essential role in modulating conflict anxiety in mice.

\section{Genetic ablation of olfactory function does not cause depression-like behavior}

The most consistent behavioral changes induced by surgical bulbectomy in mice are depression-related behaviors that can be reversed by treatment with antidepressant drugs (Harkin et al., 2003). To more accurately assess the role that olfaction plays in depression-related behaviors, we examined the consequences of genetic ablations specific to the MOE and the VNO. We used the tail suspension and the forced-swim tests (TST and FST, respectively), paradigms that test an animal's response to inescapable stress, not conflict, and are sensitive to antidepressant but not anxiolytic drugs. Using these assays, we found that the baseline behavior of Cnga2- and Trpc2-deficient mice was similar to that of controls. No significant differences were observed in the time the mice spent immobile during both assays $\left(C n a g 2^{-/ Y}\right.$ vs controls, TST, time immobile: $t_{(29)}=0.51, p>0.5$; Fig. $2 a$; FST, time immobile: $t_{(29)}=-1.59, p>0.1$; Fig. $2 b$; $\operatorname{Tr} p c 2^{-/-}$vs controls, TST, time immobile: $t_{(18)}=-0.24, p>0.5$; Fig. $2 c$; FST, time immobile: $t_{(35)}=0.04, p>0.5$; Fig. $\left.2 d\right)$. Therefore, in contrast to the surgical removal of the olfactory bulb, the genetic ablation of the MOE or VNO does not cause elevated levels of depressionrelated behaviors in the TST and FST tests. 
a Tail suspension
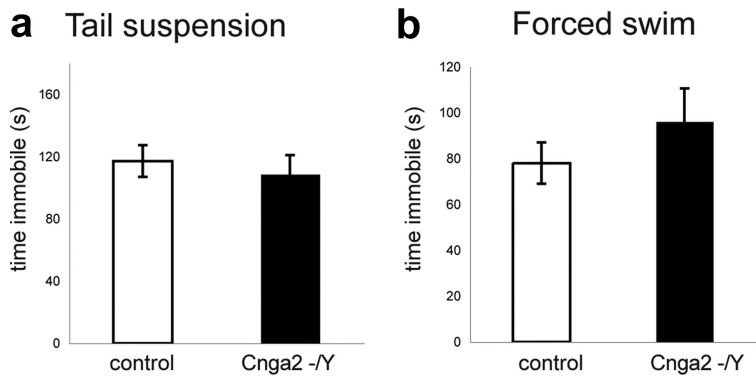

C

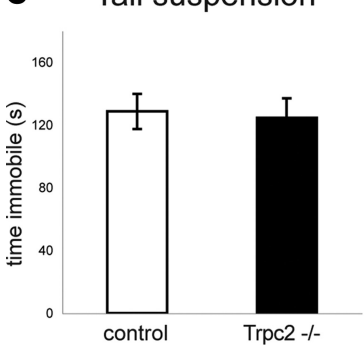

d

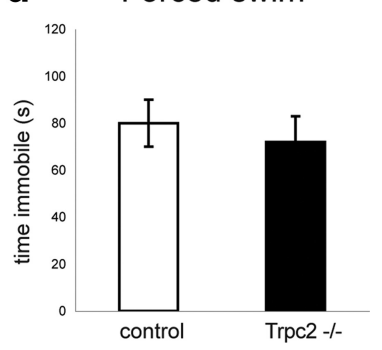

Figure 2. Lack of depression-like behaviors in $C n g a 2^{-/ \gamma}$ and $\operatorname{Trpc} 2^{-/-}$mice. $\boldsymbol{a}, \boldsymbol{b}$, No significant differences were observed in the time spent immobile in the tail suspension $(\boldsymbol{a})$ and the forced swim (b) tests between Cnga2 mutant $(n=16)$ and control $(n=15)$ mice. $\boldsymbol{c}, \boldsymbol{d}$, No significant differences were observed in the time spent immobile in the tail suspension (Trpc2 $2^{-/-}: n=11$; controls: $\left.n=9 ; c\right)$ and the forced swim (Trpc2 ${ }^{-/-}: n=22$; controls: $n=$ 15; $\boldsymbol{d})$ tests between $\operatorname{Trpc} 2$ mutant and control mice. Error bars are mean \pm SEM.

Perturbations in odor-evoked neural activity cause elevated levels of anxiety

Next, we examined the behavior of M71 transgenic (M71 Tg) mice in assays for anxiety and depression. In these mice, 95\% of all olfactory sensory neurons in the MOE and the VNO express the M71 odorant receptor, resulting in perturbations in odorevoked neural activity in the brain (Fleischmann et al., 2008), as opposed to the general anosmia of Cnga2 mutant mice. We found that M71 transgenic mice exhibited elevated levels of anxiety in the OF, LD, and NSF paradigms. In the OF test, M71 transgenic mice spent less time in the center of the arena than controls $\left(t_{(34)}=3.10, p<0.004\right.$; Fig. $\left.3 a\right)$. The percentage of distance moved in the center was significantly reduced in M71 Tg mice $\left(t_{(34)}=3.64, p<0.001\right.$; Fig. $\left.3 b\right)$, while the overall distance traveled was unchanged [path length $(\mathrm{m})$ : M71 transgenic mice: $10.5 \pm 1.2$; controls: $\left.11.1 \pm 1.0 ; t_{(34)}=0.35, p>0.7\right]$. In the LD choice test, M71 transgenic mice spent less time in the light $\left(t_{(36)}=4.17, p<0.0002\right.$; Fig. $\left.3 c\right)$. The number of light-dark transitions was similarly reduced, but this difference did not reach statistical significance (LD transitions: M71 transgenic mice: $114.1 \pm 7.8$; controls: $123.6 \pm 10.4 ; t_{(36)}=0.74, p>0.1$ ). Finally, in the NSF paradigm, the latency to feed was significantly increased in M71 transgenic mice compared with controls $\left(t_{(35)}=-2.95, p<0.006\right.$; Fig. $\left.3 d\right)$. In contrast, no behavioral changes were observed in assays for depression-related behaviors. The time spent immobile in the TST $\left(t_{(28)}=0.12, p>0.9\right.$; Fig. $\left.3 e\right)$ and in the FST $\left(t_{(31)}=1.75, p>0.09\right.$; Fig. $\left.3 f\right)$ was similar between genotypes. Furthermore, we tested whether conditioned fear responses to nonolfactory cues were altered in M71 transgenic mice. We performed classical auditory fear conditioning experiments, in which mice were exposed to three pairings of a tone with a mild foot shock. Twenty-four hours later, the mice were placed in a novel context and freezing behavior was tested. No differences were observed in the freezing behavior in the novel context or in response to the tone between M71 transgenic mice
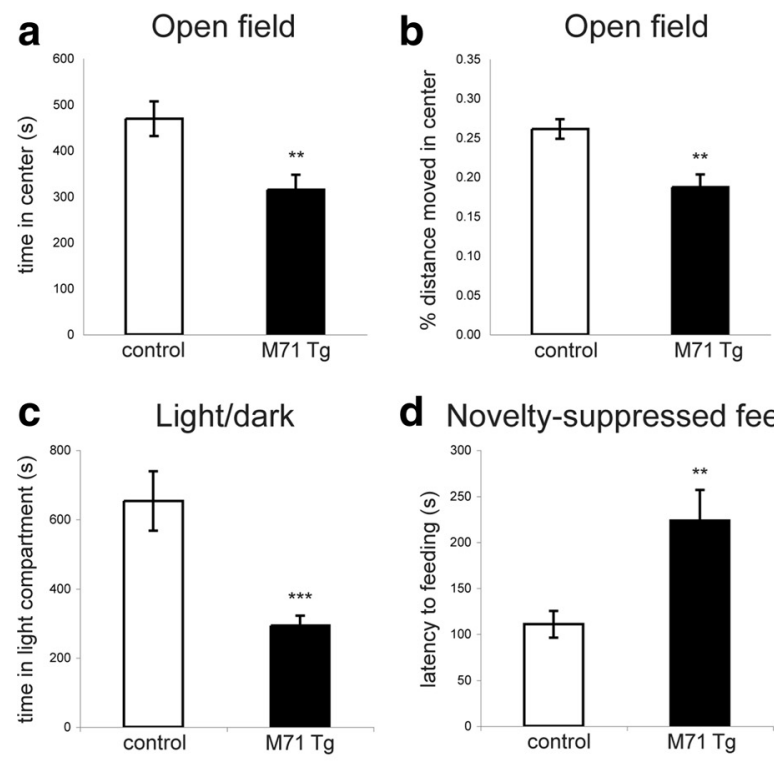

d Novelty-suppressed feeding

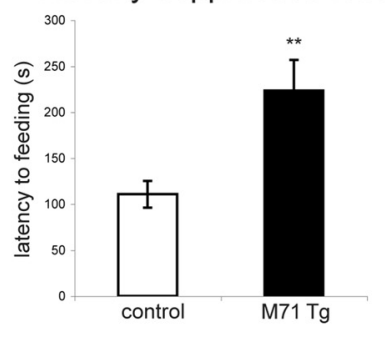

e Tail suspension f

f $\quad$ Forced swim
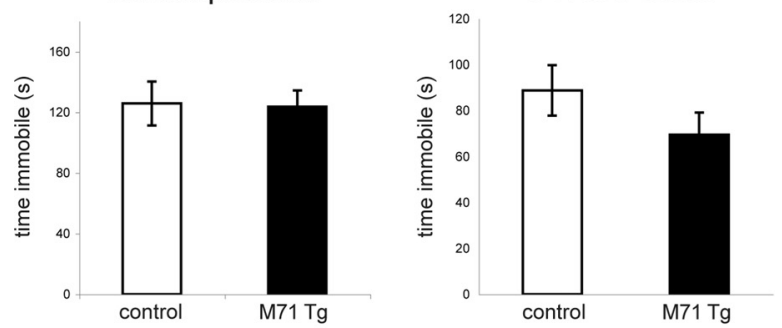

g Auditory fear conditioning

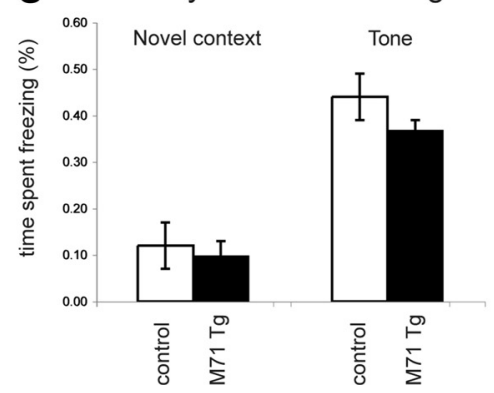

Figure 3. Elevated levels of anxiety-like behavior in M71 transgenic mice. $\boldsymbol{a}, \boldsymbol{b}$, Open field test. The time spent $(\boldsymbol{a})$ and the percentage of distance moved $(\boldsymbol{b})$ in the center of the arena is reduced in M71 transgenic ( $n=18)$ compared with control $(n=18)$ mice. c, Light-dark choice test. M71 transgenic mice spend less time in the light compartment compared with controls (M71 transgenic mice: $n=17$; controls: $n=21$ ). $\boldsymbol{d}$, Novelty-suppressed feeding. The latency to begin feeding is increased in M71 transgenic $(n=19)$ compared with control $(n=18)$ mice. $\boldsymbol{e}, \boldsymbol{f}$, Tail suspension (e) and forced swim $(\boldsymbol{f})$ tests. No significant difference was observed in the time spent immobile in the tail suspension (M71 transgenic mice: $n=16$, controls: $n=14 ; \boldsymbol{e}$ ) and the forced swim (M71 transgenic mice: $n=17$; controls: $n=16 ; \boldsymbol{f}$ ) tests between M71 transgenic and control mice. $\boldsymbol{g}$, Normal auditory fear conditioning in M71 transgenic mice. Twenty-four hours after receiving three tonefoot shock pairings, the freezing behaviors of M71 transgenic mice in a novel context and in response to the tone were similar to controls (M71 transgenic mice: $n=7$; controls: $n=7) .{ }^{* *} p<0.01,{ }^{* *} p<0.001$. Error bars are mean \pm SEM.

and controls (novel context: $t_{(12)}=0.46, p>0.6$; tone: $t_{(12)}=$ $1.38, p>0.1$; Fig. $3 g$ ), suggesting that the olfactory deficits of the M71 transgenic mice did not affect the conditioned fear response to a nonolfactory stimulus. Together, these results suggest that the perturbations in the olfactory map of M71 transgenic mice result in anxiety-like behaviors similar to, but even more pronounced than, the functional ablation of the MOE. 

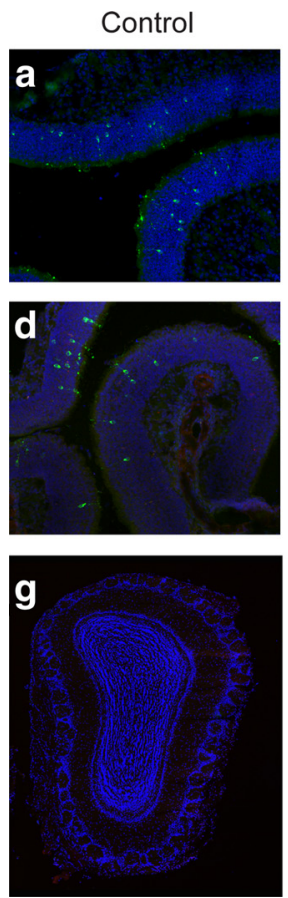

\section{M71 Tg +Dox}
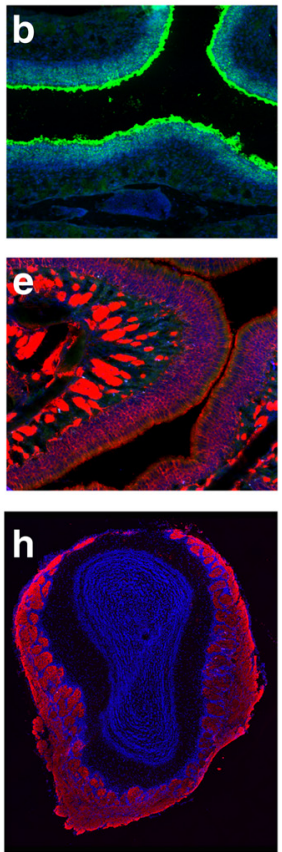
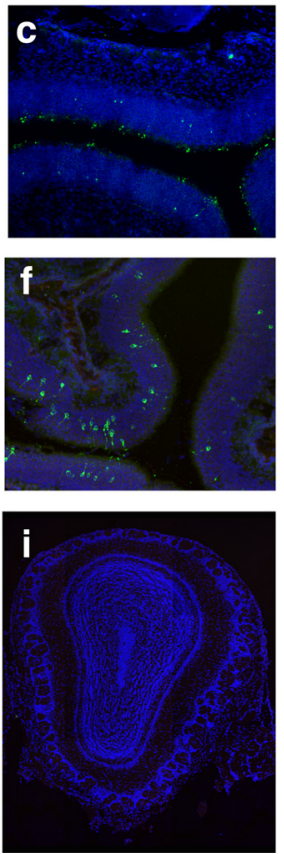

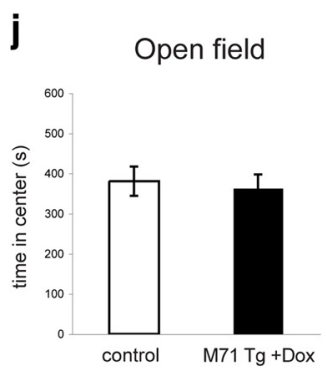

\section{I}

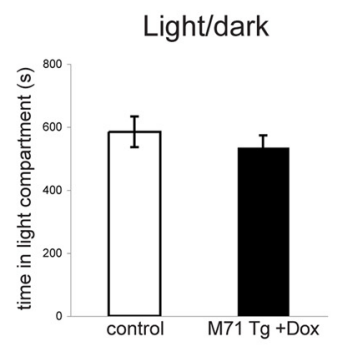

k

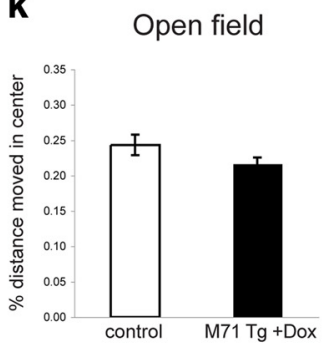

m

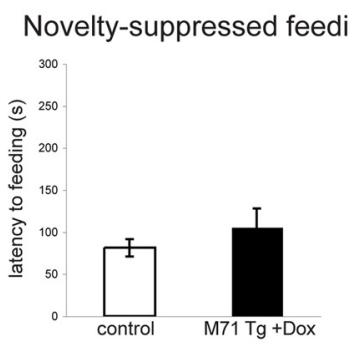

Figure 4. Dox administration suppresses M71 transgene expression and rescues the anxiety-related behaviors in M71 transgenic mice. $\boldsymbol{a}-\boldsymbol{f}$, Histological analysis of Dox-mediated transgene suppression in sections through the MOE. $\boldsymbol{a}-\boldsymbol{c}$, Immunohistochemical detection of M71 OR-expressing cells in the M0E of control (a) and M71 transgenic ( $\boldsymbol{b}$ ) mice using anti-M71 antiserum ( $\mathrm{green}$ ). c, Neurons expressing the M71 OR in M71 transgenic mice maintained on Dox-infused food. d, Immunohistochemical detection of M50 0R-expressing cells (anti-M50 antiserum; green) in the M0E of control mice. e, Detection of M71 transgene expression using anti-lacZ antiserum (red) in the M0E of M71 transgenic mice. No M50 0R-expressing neurons could be detected in M71 transgenic mice. $\boldsymbol{f}$, Detection of M50 0R-expressing neurons and suppression of M71 transgene expression in M71 transgenic mice maintained on Dox-infused food. $\boldsymbol{g}$ - $\boldsymbol{i}$, Analysis of Dox-mediated transgene suppression in coronal sections through the olfactory bulb. Expression of M71-expressing fibers visualized by anti-lacZ antiserum can be detected in M71 transgenic mice ( $h$ ), but not in control mice $(\boldsymbol{g})$ or M71 transgenic mice maintained on Dox (i). Nuclear counterstain (TOT0-3) is shown in blue. $\boldsymbol{j}-\boldsymbol{m}$, Lack of anxiety-related behaviors in M71 transgenic mice maintained on Dox. $\boldsymbol{j}, \boldsymbol{k}, 0$ pen field test (M71 transgenic mice: $n=15$; M71 transgenic mice on Dox: $n=14$ ). The time spent $(\boldsymbol{j})$ and the percentage of distance moved $(\boldsymbol{k})$ in the center of the arena is similar between M71 transgenic and control mice maintained on Dox.I, Light-dark choice test. No significant difference in the time spent in the light compartment was observed between M71 transgenic mice ( $n=17$ ) and controls $(n=13)$ maintained on Dox. $\boldsymbol{m}$, Novelty-suppressed feeding. The latency to begin feeding was similar between M71 transgenic mice $(n=18)$ and controls ( $n=13)$ maintained on Dox. Error bars are mean \pm SEM.

\section{Doxycycline-mediated suppression of M71 expression rescues the anxiety-like behavior}

To ascertain whether the anxiety-related behaviors of M71 transgenic mice are directly caused by their olfactory deficits, and to exclude the possibility that the genetic alterations at the transgene insertion site could affect their behaviors independent of the pervasive expression of the M71 odorant receptor, we took advantage of the doxycycline-dependent control of transgene expression. Dox ablates the DNA binding of the tTA transcription factor to its cognate binding sites in the promoter and shuts off transgene expression (Gossen et al., 1995). We administered Dox-infused food to M71 transgenic and control mice during development (through maternal feeding) and maintained the mice on Dox until 12 weeks of age. To examine the effect of Dox on M71 transgene expression, we then processed the MOE and the olfactory bulb for histological analysis. Individual ORs are expressed in $\sim 0.1 \%$ of the sensory neurons in the MOE of wild-type mice, exemplified by immunohistochemical staining of M71 and M50 OR-expressing cells (Fig. 4a,d). In contrast, the expression of the M71 OR in the vast majority of neurons suppresses endogenous OR gene expression (Fig. 4b,e). We found that in the presence of Dox, M71 transgene expression was completely abolished, as shown by the absence of lacZ-positive sensory neurons in the MOE. Moreover, the frequency of neurons expressing the endogenous M71 and M50 OR was indistinguishable from controls (Fig. 4c,f). Similarly, the suppression of M71 transgene expression by Dox was evident in the olfactory bulb. In M71 transgenic mice, M71-expressing fibers innervated all glomeruli
(Fig. 4h). In contrast, in the presence of Dox, the olfactory bulbs of M71 transgenic mice were devoid of M71-expressing fibers (Fig. 4i). Thus, Dox administration resulted in the efficient suppression of M71 OR expression. If pervasive M71 OR expression and the resulting perturbations in odor-evoked neural activity are solely responsible for the elevated levels of anxiety in M71 transgenic mice, the administration of Dox should rescue this behavioral deficit. Indeed, we observed that the anxiety-related behaviors of M71 transgenic mice maintained on Dox were indistinguishable from those of controls. No significant differences were observed for anxiety-related behaviors in the OF test (time spent in the center of the arena: $t_{(27)}=$ $0.54, p>0.5$; Fig. $4 j$; percentage of distance moved in the center: $t_{(27)}$ $=0.40, p>0.6$; Fig. $4 k$ ). Similarly, the time spent in the light compartment of the LD test was similar between M71 transgenic mice on Dox and controls $\left(t_{(28)}=-1.84, p>0.07\right.$; Fig. $\left.4 l\right)$. Finally, no difference was observed in the latency to begin feeding in the NSF test $\left(t_{(29)}=-0.31, p>0.7\right.$; Fig. $\left.4 m\right)$. These results strongly support the conclusion that the expression of a single OR in the vast majority of olfactory sensory neurons, and the resulting perturbations in odor-evoked neural activity, directly cause the elevated levels of anxiety observed in M71 transgenic mice.

\section{Perturbations in odor-evoked neural activity cause elevated blood corticosterone levels}

How could olfactory deficits cause changes in anxiety-related behaviors in approach-avoidance tasks? One possibility is that mice predominantly rely on odor cues to direct their behavior during 
these tests. Olfactory deficits in this scenario would lead to acutely elevated levels of anxiety. Another possibility is that the lack or the inaccurate detection of olfactory information results in more generalized deficits in the ability of mice to probe their environment. Such deficits could cause chronic stress, resulting in persistently elevated levels of anxiety (Koike et al., 2009). To discriminate between these possibilities, we first examined whether exposure of mice to the OF test would predominantly activate olfactory centers in the brain. We placed mice into the $\mathrm{OF}$ and, after $45 \mathrm{~min}$, processed brains for immunohistochemical analysis of c-Fos expression. Activation of the immediate-early gene product $\mathrm{c}$-Fos can result from elevations in intracellular calcium and is often used as a marker of neural activity (Morgan and Curran, 1991). We found that compared with mice in their home cage, exposure to the OF arena resulted in the induction of c-Fos immunoreactivity in multiple sensory areas in the brain, including the olfactory (piriform), visual, and somatosensory cortices (Fig. $5 a-e$ ). We quantified the number of c-Fos-positive cells in the piriform cortex (Pir), V1, and S1 and found that, compared with home cage controls, the number of c-Fos-positive cells was robustly increased. In wild-type mice, the number of c-Fos-positive cells in piriform cortex was increased $\sim 10$-fold [c-Fos-positive nuclei per region of interest (Pir), home cage: $4.2 \pm 3.0$; OF: $37.6 \pm 10.8$; Fig. 5e]. In visual and somatosensory cortices, activation of c-Fos expression was even more pronounced, resulting in a $>100$-fold increase in c-Fos-positive nuclei. (c-Fos-positive nuclei in V1, home cage: $0.3 \pm 0.2$; OF: $83.3 \pm 28.2$; c-Fos-positive nuclei in S1, home cage: $0.6 \pm 0.4$; OF: $72.8 \pm 15.3$; Fig. $5 e$ ). Importantly, the observed increase in c-Fos expression was similar between control and M71 transgenic mice in piriform cortex, V1, and S1 (M71 transgenic mice, c-Fospositive nuclei in piriform cortex, home cage: $5.0 \pm 2.7$; OF: $32.2 \pm 7.0$; V1, home cage: $2.0 \pm 0.8$; OF: $70.9 \pm 19.3$; S1, home cage: $2.4 \pm 1.2$; OF: $73.5 \pm 15.2$; Fig. $5 e$ ). These results suggest that exposure to the OF test results in the activation of multiple sensory areas in the cortex. Therefore, olfactory cues are unlikely to be the only source of sensory information used by mice during this test.

We next considered the possibility that olfactory deficits could cause chronic stress. Chronic stress can cause elevated levels of anxiety and is often correlated with an increase in blood glucocorticoid levels (Müller et al., 2003). We therefore measured the plasma corticosterone levels under baseline (home cage) conditions and found that corticosterone levels were approximately twofold increased in M71 transgenic mice compared with controls (M71 Tg: 16,251 \pm 1219 pg/ml; controls: $8992 \pm 1292$ pg/ $\mathrm{ml}, t_{(30)}=-2.44, p<0.05$; Fig. $5 f$ ). As expected, corticosterone levels were further increased upon exposure of mice to the OF test. Corticosterone levels in M71 transgenic remained elevated compared with controls, but this difference was not statistically significant (data not shown). In contrast, corticosterone levels in $\operatorname{Trp} c 2$ mutant mice were unaltered $\left(\operatorname{Trpc} 2^{-/-}: 8672 \pm 1866 \mathrm{pg} /\right.$ $\mathrm{ml}$, controls: $\left.9542 \pm 1513 \mathrm{pg} / \mathrm{ml} n=7 ; t_{(13)}=0.36, p>0.5\right)$, consistent with the absence of anxiety-like behaviors. We did not measure blood corticosterone levels in Cnga2 knock-out mice, due to the high incidence of postnatal lethality and the consequent difficulties in obtaining a large enough cohort of mice. Our data reveal that the perturbations in odor-evoked neural activity in M71 transgenic mice can lead to elevated plasma corticosterone levels, consistent with chronic stress and the elevated levels of anxiety.
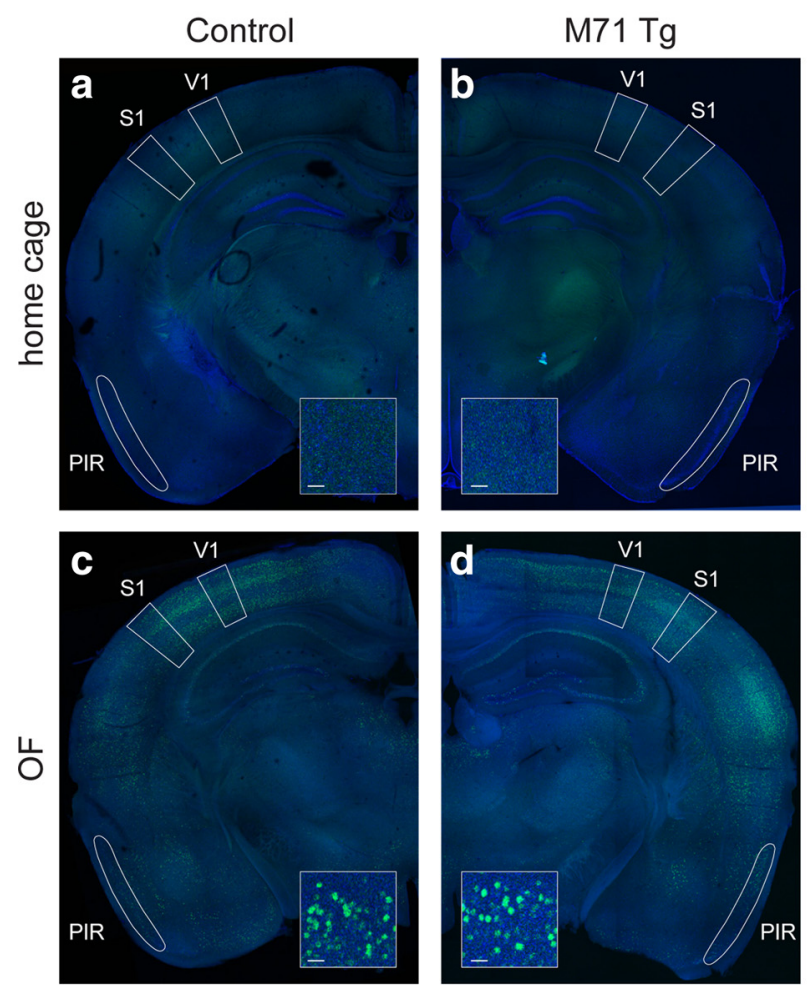

e

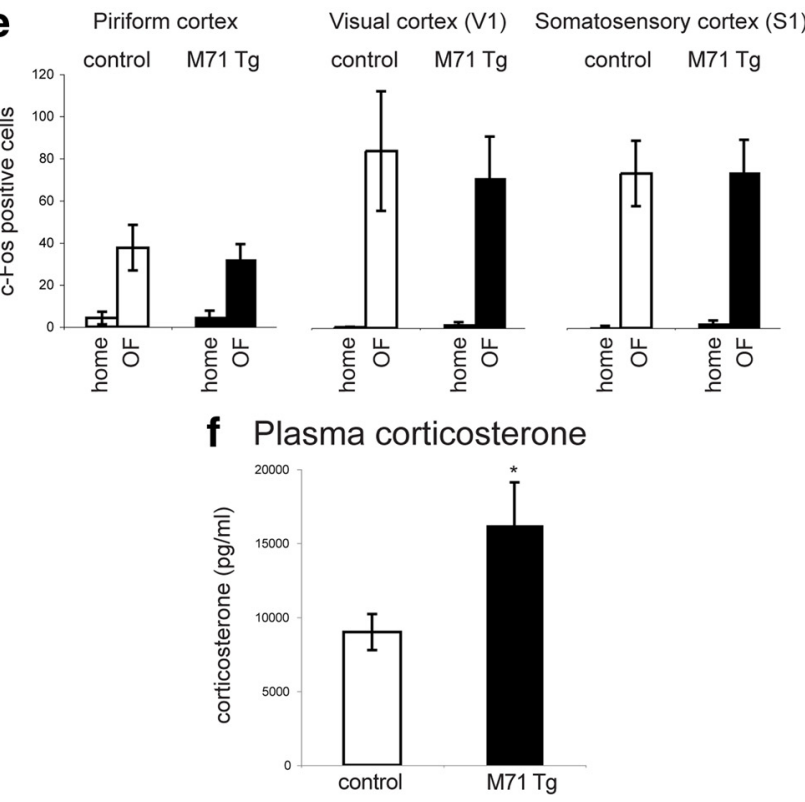

Figure 5. Activation of multiple sensory areas in the cortex during the OF test and elevated plasma corticosterone levels in M71 transgenic mice. $\boldsymbol{a}-\boldsymbol{d}$, Immunohistochemical analysis of c-Fos expression under baseline (home cage) conditions and after exposure to the OF test in control $(\boldsymbol{a}, \boldsymbol{c})$ and M71 transgenic $(\boldsymbol{b}, \boldsymbol{d})$ mice. A robust increase in c-Fos activity upon OF exposure is observed in multiple brain areas, including $\mathrm{V} 1$ and $\boldsymbol{S 1}(\boldsymbol{b}, \boldsymbol{d})$. A moderate increase in c-Fos activity is observed in PIR. Anti-c-Fos immunoreactivity is shown in green, NeuroTrace counterstain in blue. Scale bar, $10 \mu \mathrm{m}$. $\boldsymbol{e}$, Quantification of c-Fos-positive nuclei in piriform cortex, V1, and S1, as defined by the boxed areas in $\boldsymbol{a}-\boldsymbol{d}$. Similar induction of c-Fos immunoreactivity was observed in M71 transgenic and control mice. $f$, Elevated levels of plasma corticosterone in M71 transgenic mice compared with controls under baseline (home cage) conditions (M71 transgenic mice: $n=15$; controls: $n=17$ ). ${ }^{*} p<0.05$. Error bars are mean \pm SEM. 


\section{Discussion}

Our results suggest that olfactory cues are essential for modulating conflict anxiety in mice. Previously, a large number of lesion studies found complex behavioral changes upon surgical removal of the olfactory bulbs, including hyperactivity, irritability, and passive avoidance deficits. These changes were interpreted as depression-like behaviors and were alleviated by chronic antidepressant treatment (Cairncross et al., 1977; van Riezen and Leonard, 1990; Brunjes, 1992; Kelly et al., 1997; Harkin et al., 2003; Song and Leonard, 2005). Surgical ablations, however, are complicated by the variability of the lesion and by widespread secondary effects, including axonal degeneration, regeneration, inflammation; and neurochemical and hormonal changes (Brunjes, 1992; Harkin et al., 2003; Song and Leonard, 2005). Here, we have analyzed genetically modified mouse lines with a highly specific functional ablation of main olfactory function and perturbations in odor-evoked neural activity, and we demonstrate a resultant increase in the levels of anxiety-related behaviors in the OF, LD, and NSF paradigms. In contrast, depression-like behaviors in the TST and FST tests were not increased. One interpretation consistent with our results is that perturbations in olfactory function directly affect anxiety-like behaviors, while surgical removal of the olfactory bulbs results in additional behavioral changes secondary to the olfactory deficits.

The targeted mutations in the Cnga2 and $\operatorname{Trpc} 2$ genes provide an opportunity to determine the relative contributions of the $\mathrm{MOE}$ and the VNO to the behavioral phenotypes. Increased anxiety was observed in Cnga2-deficient, but not in $\operatorname{Trpc2}$-deficient, mice. Thus, olfactory cues detected by the MOE, but not the VNO, modulate anxiety. This finding may be surprising in the light of strong connections between the accessory olfactory system and limbic structures involved in the control of mood and fear (Dulac and Wagner, 2006). However, neural substrates implicated in the control of anxiety, such as the central nucleus of the amygdala and the ventral hippocampus (Shin and Liberzon, 2010; Tye et al., 2011), have been shown to receive strong sensory input from the main olfactory pathway (Schwabe et al., 2004; Kent et al., 2007). Furthermore, the MOE has been demonstrated to mediate important social behaviors in mice, which strongly impact on the function of the limbic system (Restrepo et al., 2004; Mandiyan et al., 2005).

In M71 transgenic mice, the patterns of odor-evoked neural activity are perturbed. Exposure to acetophenone, an M71 receptor ligand, results in a massive increase in neural activity in the MOE and the olfactory bulb, while odorants that do not activate the M71 receptor fail to elicit detectable neural responses (Fleischmann et al., 2008). We found that the perturbations in olfactory sensory input in M71 transgenic mice also cause anxiety-like behaviors, while depression-like behaviors and auditory fear conditioning were unaffected. The anxiety-like behaviors of M71 transgenic mice were similar to, but even more pronounced, than those observed in mice with a functional ablation of the MOE. These elevated levels of anxiety are likely a direct consequence of alterations in odor-evoked neural activity, since administration of doxycycline during development results in the suppression of M71 transgene expression and normal behaviors in approach-avoidance conflict tasks. The M71 OR has been shown to respond to acetophenone, a compound present in mouse urine (Lin et al., 2005). It is possible that the continuous exposure of mice to this odorant, and the consequent continuous global activation of olfactory bulb, may underlie the development of anxiety in these mice. However, Cnga2 mutant mice, which are incapable of detecting odors, show similar behavioral phenotypes. We therefore favor the model that the observed anxiety behaviors are a consequence of general deficit in olfactory detection and processing rather than reflecting specific anxietyinducing functions of either the Cnga2 or the M71 odorant receptor genes. The results further suggest that altered olfactory signals may provide conflicting or inaccurate sensory information, resulting in more severe behavioral deficits than the complete loss of olfactory perception.

Are approach-avoidance conflict tasks predominantly olfactory-driven behavioral tests? The behavior of mice in tests for anxiety-like behaviors, including the OF test, is sensitive to multiple sensory cues, including visual and somatosensory cues. For example, the anxiety-related parameters of the behavior of mice in the OF test are sensitive to the intensity of illumination of the arena (Crawley, 1985). In contrast, it is unknown whether mice use olfactory information to direct their behaviors during the OF test and preliminary experiments with odors placed into the open field arena failed to yield robust effects on anxietyrelated parameters of behavior (M. E. Glinka and A. Fleischmann, unpublished observations). Assuming that the olfactory environment is homogeneous across the arena, it therefore appears unlikely that odor cues dominate over visual or somatosensory information during the test.

Consistent with the idea that the OF test is a multisensory task, we observe that visual and somatosensory areas in the cortex are robustly activated during the OF test. The frequency of neurons expressing the immediate-early gene product c-Fos, a well characterized marker for neural activity, is increased $>100$-fold in V1 and S1. A less dramatic increase $(\sim 10$-fold $)$ in c-Fos-positive neurons is observed in the olfactory (piriform) cortex. Importantly, no differences were observed in the induction of c-Fos activity in primary olfactory, visual, or somatosensory cortices between M71 transgenic mice and controls. Therefore, an alternative explanation for the elevated levels of anxiety in M71 transgenic mice could be that altered olfactory signals provide inaccurate sensory information, resulting in chronic stress. Olfactory cues have been shown to be essential for normal social behaviors (Restrepo et al., 2004; Mandiyan et al., 2005; Spehr et al., 2006), and M71 transgenic mice have severe deficits in sexual and aggressive behaviors (Fleischmann et al., 2008). It is possible that the inability to correctly interpret social odor cues may result in a scenario similar to social isolation. Animals reared in isolation provide a well understood paradigm for investigating the effects of early-life stress and for understanding the pathogenesis of neurological and psychiatric disease (Myhrer, 1998; WhitakerAzmitia et al., 2000; Ibi et al., 2008). Changes induced by social isolation in rodents are widespread and can be detected at behavioral (Lu et al., 2003; Day-Wilson et al., 2006), neurochemical (Heidbreder et al., 2000; Muchimapura et al., 2003), and histological (Silva-Gómez et al., 2003; Ibi et al., 2008; Dranovsky et al., 2011) levels of analysis. In addition, social isolation, like several other stressors, is capable of yielding elevated levels of corticosterone (Bowers et al., 2008). This model is consistent with our observation that even under baseline (home cage) conditions, M71 transgenic mice exhibit an approximately twofold increase in the levels of blood plasma corticosterone, a physiological marker associated with chronic stress.

Together, our data show that genetic ablation and perturbation of olfactory function in mice can cause complex behavioral changes not previously considered a consequence of anosmia. Such sensory deficits likely affect the function of neural circuits involved in the control of mood and fear, a 
scenario that may be relevant to the development of human anxiety disorders.

\section{References}

Bowers SL, Bilbo SD, Dhabhar FS, Nelson RJ (2008) Stressor-specific alterations in corticosterone and immune responses in mice. Brain Behav Immun 22:105-113.

Brunet LJ, Gold GH, Ngai J (1996) General anosmia caused by a targeted disruption of the mouse olfactory cyclic nucleotide-gated cation channel. Neuron 17:681-693.

Brunjes PC (1992) Lessons from lesions: the effects of olfactory bulbectomy. Chem Senses 17:729-763.

Buck L, Axel R (1991) A novel multigene family may encode odorant receptors: a molecular basis for odor recognition. Cell 65:175-187.

Cairncross KD, Cox B, Forster C, Wren AF (1977) The olfactory bulbectomized rat: a simple model for detecting drugs with antidepressant potential [proceedings]. Br J Pharmacol 61:497P.

Crawley JN (1985) Exploratory behavior models of anxiety in mice. Neurosci Biobehav Rev 9:37-44.

David DJ, Samuels BA, Rainer Q, Wang JW, Marsteller D, Mendez I, Drew M, Craig DA, Guiard BP, Guilloux JP, Artymyshyn RP, Gardier AM, Gerald C, Antonijevic IA, Leonardo ED, Hen R (2009) Neurogenesisdependent and -independent effects of fluoxetine in an animal model of anxiety/depression. Neuron 62:479-493.

Day-Wilson KM, Jones DN, Southam E, Cilia J, Totterdell S (2006) Medial prefrontal cortex volume loss in rats with isolation rearing-induced deficits in prepulse inhibition of acoustic startle. Neuroscience 141:11131121.

Dranovsky A, Picchini AM, Moadel T, Sisti AC, Yamada A, Kimura S, Leonardo ED, Hen R (2011) Experience dictates stem cell fate in the adult hippocampus. Neuron 70:908-923.

Dulac C, Wagner S (2006) Genetic analysis of brain circuits underlying pheromone signaling. Annu Rev Genet 40:449-467.

Fleischmann A, Shykind BM, Sosulski DL, Franks KM, Glinka ME, Mei DF, Sun Y, Kirkland J, Mendelsohn M, Albers MW, Axel R (2008) Mice with a "monoclonal nose": perturbations in an olfactory map impair odor discrimination. Neuron 60:1068-1081.

Franklin KB, Paxinos G (2007) The mouse brain atlas, third edition. San Diego: Academic.

Gossen M, Freundlieb S, Bender G, Müller G, Hillen W, Bujard H (1995) Transcriptional activation by tetracyclines in mammalian cells. Science 268:1766-1769.

Harkin A, Kelly JP, Leonard BE (2003) A review of the relevance and validity of olfactory bulbectomy as a model of depression. Clin Neurosci Res 3:253-262.

Heidbreder CA, Weiss IC, Domeney AM, Pryce C, Homberg J, Hedou G, Feldon J, Moran MC, Nelson P (2000) Behavioral, neurochemical and endocrinological characterization of the early social isolation syndrome. Neuroscience 100:749-768.

Ibi D, Takuma K, Koike H, Mizoguchi H, Tsuritani K, Kuwahara Y, Kamei H, Nagai T, Yoneda Y, Nabeshima T, Yamada K (2008) Social isolation rearing-induced impairment of the hippocampal neurogenesis is associated with deficits in spatial memory and emotion-related behaviors in juvenile mice. J Neurochem 105:921-932.

Kelly JP, Wrynn AS, Leonard BE (1997) The olfactory bulbectomized rat as a model of depression: an update. Pharmacol Ther 74:299-316.

Kent K, Hess K, Tonegawa S, Small SA (2007) CA3 NMDA receptors are required for experience-dependent shifts in hippocampal activity. Hippocampus 17:1003-1011.

Kessler RC, Ruscio AM, Shear K, Wittchen HU (2010) Epidemiology of anxiety disorders. Curr Top Behav Neurosci 2:21-35.

Kimchi T, Xu J, Dulac C (2007) A functional circuit underlying male sexual behaviour in the female mouse brain. Nature 448:1009-1014.

Koike H, Ibi D, Mizoguchi H, Nagai T, Nitta A, Takuma K, Nabeshima T, Yoneda Y, Yamada K (2009) Behavioral abnormality and pharmaco- logic response in social isolation-reared mice. Behav Brain Res 202:114-121.

Leypold BG, Yu CR, Leinders-Zufall T, Kim MM, Zufall F, Axel R (2002) Altered sexual and social behaviors in trp2 mutant mice. Proc Natl Acad Sci U S A 99:6376-6381.

Lin DY, Zhang SZ, Block E, Katz LC (2005) Encoding social signals in the mouse main olfactory bulb. Nature 434:470-477.

Lu L, Bao G, Chen H, Xia P, Fan X, Zhang J, Pei G, Ma L (2003) Modification of hippocampal neurogenesis and neuroplasticity by social environments. Exp Neurol 183:600-609.

Mandiyan VS, Coats JK, Shah NM (2005) Deficits in sexual and aggressive behaviors in Cnga2 mutant mice. Nat Neurosci 8:1660-1662.

Morgan JI, Curran T (1991) Stimulus-transcription coupling in the nervous system: involvement of the inducible proto-oncogenes fos and jun. Annu Rev Neurosci 14:421-451.

Muchimapura S, Mason R, Marsden CA (2003) Effect of isolation rearing on pre- and post-synaptic serotonergic function in the rat dorsal hippocampus. Synapse 47:209-217.

Müller MB, Zimmermann S, Sillaber I, Hagemeyer TP, Deussing JM, Timpl P, Kormann MS, Droste SK, Kühn R, Reul JM, Holsboer F, Wurst W (2003) Limbic corticotropin-releasing hormone receptor 1 mediates anxiety-related behavior and hormonal adaptation to stress. Nat Neurosci 6:1100-1107.

Myhrer T (1998) Adverse psychological impact, glutamatergic dysfunction, and risk factors for Alzheimer's disease. Neurosci Biobehav Rev 23: $131-139$.

Nguyen MQ, Zhou Z, Marks CA, Ryba NJ, Belluscio L (2007) Prominent roles for odorant receptor coding sequences in allelic exclusion. Cell 131:1009-1017.

Nguyen MQ, Marks CA, Belluscio L, Ryba NJ (2010) Early expression of odorant receptors distorts the olfactory circuitry. J Neurosci 30:92719279.

Restrepo D, Arellano J, Oliva AM, Schaefer ML, Lin W (2004) Emerging views on the distinct but related roles of the main and accessory olfactory systems in responsiveness to chemosensory signals in mice. Horm Behav 46:247-256

Schwabe K, Ebert U, Löscher W (2004) The central piriform cortex: anatomical connections and anticonvulsant effect of GABA elevation in the kindling model. Neuroscience 126:727-741.

Shin LM, Liberzon I (2010) The neurocircuitry of fear, stress, and anxiety disorders. Neuropsychopharmacology 35:169-191.

Silva-Gómez AB, Rojas D, Juárez I, Flores G (2003) Decreased dendritic spine density on prefrontal cortical and hippocampal pyramidal neurons in postweaning social isolation rats. Brain Res 983:128-136.

Song C, Leonard BE (2005) The olfactory bulbectomised rat as a model of depression. Neurosci Biobehav Rev 29:627-647.

Spehr M, Kelliher KR, Li XH, Boehm T, Leinders-Zufall T, Zufall F (2006) Essential role of the main olfactory system in social recognition of major histocompatibility complex peptide ligands. J Neurosci 26:1961-1970.

Stowers L, Holy TE, Meister M, Dulac C, Koentges G (2002) Loss of sex discrimination and male-male aggression in mice deficient for TRP2. Science 295:1493-1500.

Tye KM, Prakash R, Kim SY, Fenno LE, Grosenick L, Zarabi H, Thompson KR, Gradinaru V, Ramakrishnan C, Deisseroth K (2011) Amygdala circuitry mediating reversible and bidirectional control of anxiety. Nature 471:358-362.

van Riezen H, Leonard BE (1990) Effects of psychotropic drugs on the behavior and neurochemistry of olfactory bulbectomized rats. Pharmacol Ther 47:21-34.

Whitaker-Azmitia P, Zhou F, Hobin J, Borella A (2000) Isolation-rearing of rats produces deficits as adults in the serotonergic innervation of hippocampus. Peptides 21:1755-1759.

Zhang X, Firestein S (2002) The olfactory receptor gene superfamily of the mouse. Nat Neurosci 5:124-133. 